OPEN ACCESS

Edited by:

Christopher Butler

University of Oxford, United Kingdom

Reviewed by:

Anthoula Charalampos Tsolaki, Aristotle University of Thessaloniki,

Greece

Victoria Susan Pelak,

University of Colorado Denver,

United States

${ }^{*}$ Correspondence:

Qun Xu

xuqun628@hotmail.com

Yan Zhou

clare1475@hotmail.com

tThese authors have contributed equally to this work

Specialty section:

This article was submitted to

Dementia,

a section of the journal

Frontiers in Neurology

Received: 13 December 2018

Accepted: 21 March 2019

Published: 12 April 2019

Citation:

Wu X, Ge X, Du J, Wang Y, Sun Y, Han $X$, Ding W, Cao $M, X u Q$ and Zhou Y (2019) Characterizing the

Penumbras of White Matter Hyperintensities and Their Associations With Cognitive Function in Patients With Subcortical Vascular Mild Cognitive Impairment

Front. Neurol. 10:348

doi: 10.3389/fneur.2019.00348

\section{Characterizing the Penumbras of White Matter Hyperintensities and Their Associations With Cognitive Function in Patients With Subcortical Vascular Mild Cognitive Impairment}

\author{
Xiaowei Wu ${ }^{1 \dagger}$, Xin Ge ${ }^{1+}$, Jing $\mathrm{Du}^{2}$, Yao Wang ${ }^{1}$, Yawen Sun ${ }^{1}$, Xu Han ${ }^{1}$, Weina Ding ${ }^{1}$, \\ Mengqiu $\mathrm{Cao}^{1}$, Qun $\mathrm{Xu}^{2 *}$ and Yan Zhou ${ }^{1 *}$ \\ ${ }^{1}$ Department of Radiology, Ren Ji Hospital, School of Medicine, Shanghai Jiao Tong University, Shanghai, China, \\ ${ }^{2}$ Department of Neurology, Ren Ji Hospital, School of Medicine, Shanghai Jiao Tong University, Shanghai, China
}

Normal-appearing white matter (NAWM) surrounding white matter hyperintensities (WMHs), frequently known as the WMH penumbra, is associated with subtle white matter injury and has a high risk for future conversion to WMHs. The goal of this study was to define WMH penumbras and to further explore whether the diffusion and perfusion parameters of these penumbras could better reflect cognitive function alterations than WMHs in subjects with subcortical vascular mild cognitive impairment (svMCl). Seventy-three svMCl subjects underwent neuropsychological assessments and 3T MRI scans, including diffusion tensor imaging (DTI) and arterial spin labeling (ASL). To determine the extent of cerebral blood flow (CBF) and DTI penumbras. A NAWM layer mask was generated for periventricular WMHs (PWWMHs) and deep WMHs (DWMHs) separately. Mean values of CBF, fractional anisotropy (FA), mean diffusivity (MD) within the WMHs and their corresponding NAWM layer masks were computed and compared using paired $t$-tests. Pearson's partial correlations were used to assess the relations of the mean $\mathrm{CBF}, \mathrm{FA}$, and MD values within the corresponding penumbras with composite z-scores of global cognition and four cognitive domains controlling for age, sex, and education. For both PVWMHs and DWMHs, the CBF penumbras were wider than the DTI penumbras. Only the mean FA value of the PWWMH-FA penumbra was correlated with the composite $z$-scores of global cognition before correction $(r=0.268, p=0.024)$, but that correlation did not survive after correcting the $p$-value for multiple comparisons. Our findings showed extensive white matter perfusion disturbances including white matter tissue, both with and without microstructural alterations. The imaging parameters investigated, however, did not correlate to cognition.

\footnotetext{
Keywords: white matter hyperintensity, normal appearing white matter, penumbra, cerebral blood flow, diffusion tensor imaging, subcortical vascular mild cognitive impairment
} 


\section{INTRODUCTION}

Vascular cognitive impairment (VCI) refers to all levels of cognitive alteration, ranging from mild to severe, caused by cerebrovascular disease (1). Subcortical vascular cognitive impairment (SVCI) is a common form of VCI caused by subcortical ischemic vascular disease (SIVD) (2). Subcortical vascular mild cognitive impairment (svMCI) is a prodromal stage of Subcortical vascular dementia (SVaD) (3). It is clinically important to focus on svMCI before it develops into SVaD. White matter hyperintensities (WMHs), also known as WM lesions or leukoaraiosis, are usually considered the most common magnetic resonance imaging (MRI) manifestations of SIVD, increasing with both age and vascular risk factors $(4,5)$. WMHs are frequently divided into periventricular WMH (PVWMH) and deep WMH (DWMH), and the two classifications have been differentially associated with vascular risk factors (6-9), cognitive function measures (10-14) and histopathologic findings (1417). The burden of WMHs has widely been reported to be associated with cognitive decline and the progression of cognitive impairment $(13,18-22)$.

There is an increasing amount of evidence suggesting that WM alterations are not only present in visible WMHs but also in the normal-appearing WM (NAWM) surrounding WMHs, which conventional MRI scans re unable to identify. Previous reports have indicated that NAWM displays subtle immunohistochemical and pathological alterations beyond WM lesions, raising the possibility that WM dysfunction is more widespread than the focal WM lesions (23-26). Previous studies have also demonstrated WM integrity disruption and hypoperfusion within NAWM surrounding WMHs, using diffusion tensor imaging (DTI) and arterial spin labeling (ASL) (27-31). The specific, subtly changed NAWM surrounding WMHs is called a "WMH penumbra" in many studies; however, this terminology has not yet been widely adopted.

WMH penumbras represent milder WM injury concerning WMHs and are at a higher risk for future conversion to WMH than other areas of healthy WM beyond penumbras (29). Previous studies have shown that structural WMH penumbras, defined by DTI and FLAIR intensity, extended approximately $2-9 \mathrm{~mm}$, while cerebral blood flow (CBF) WMH penumbras, defined by ASL, extended $\sim 12-14 \mathrm{~mm}$ around both PVWMH and DWMH in cognitively intact communitydwelling elderly individuals $(27-29,32)$. Though the extent of WMH penumbras is not entirely consistent among studies, these findings consistently reveal that $\mathrm{CBF}$ penumbras are more extensive than structural penumbras, indicating that altered CBF may predate structural changes in the NAWM surrounding WMH. Longitudinal studies have further demonstrated that WMHs and their penumbras represent a continuum of WM injury that evolves over time and that abnormal changes in surrounding NAWM precede the progression of WMH (27, $28,32,33)$. Other studies have also found that most of the new lacunes preferentially localize to $\mathrm{WMH}$ penumbras and that infarcts on the edge of WMHs are more likely to develop into lacunes or cavities than those far from WMHs $(34,35)$. These findings imply that WMH penumbras can be regarded as relevant clinical targets for interventions that prevent the development of WMHs. Structural penumbras defined by DTI may provide information about full-scale microstructural WM integrity disruption, and CBF penumbras may provide etiological insight into the formation and progression of WMHs. Therefore, it is crucial to combine structural and $\mathrm{CBF}$ penumbras of WMHs within svMCI patients to facilitate our understanding of the mechanism underlying WMH progression and cognitive impairment evolution.

Currently, studies characterizing both structural and CBF WMH penumbra in the same svMCI subjects are lacking. The goal of this study is to characterize the $\mathrm{CBF}$ and structural penumbras of PVWMHs and DWMHs and to further explore whether penumbras can reflect cognitive function alterations, better than WMHs themselves, among svMCI subjects.

\section{MATERIALS AND METHODS}

\section{Subjects}

SIVD subjects were recruited from patients who were admitted to the Neurology Department of Ren Ji Hospital between August 2015 and December 2017. SIVD was defined as a subcortical WM hyperintensity on T2-FLAIR imaging with at least one lacunar infarct, according to the criteria suggested by Galluzzi et al. (36). SIVD subjects who fulfilled svMCI criteria, suggested by Petersen et al. (37) and Gorelick et al. (38), were included in our study. The inclusion criteria were as follows: (1) subjective cognitive difficulty reported by the patient or caregiver; (2) quantifiable cognitive decline within one or more cognitive domains (e.g., attention-executive function, memory, language, or visuospatial function); (3) normal instrumental activity of daily living. The exclusion criteria were as follows: (1) cortical and/or corticosubcortical non-lacunar territorial infarcts and watershed infarcts; (2) neurodegenerative diseases (including Parkinson's disease and AD); (3) signs of normalpressure hydrocephalus; (4) specific causes of WM lesions (e.g., metabolic, toxic, infectious, multiple sclerosis, brain irradiation); (5) alcoholic encephalopathy or illicit drug use; (6) major depression (Hamilton Depression Rating Scale (HDRS) $\geq 18$ ) (39); (7) severe cognitive impairment (inability to perform the neuropsychological test or undergo the whole MRI scan); (8) MRI safety contraindications and claustrophobia; (9) education $\leq 6$ years. Early VCI is characterized by executive function/processing speed deficits with relatively preserved memory and is less likely to produce subjective complaints, whereas Alzheimer's disease (AD) or mixed cognitive impairment feature memory problems (2). Thus, we carefully excluded the participants with memory complaints. All patients underwent laboratory examinations to exclude systemic or other neurological diseases. Finally, 73 right-handed svMCI patients were included in this study.

The present research was approved by the Research Ethics Committee of the Ren Ji Hospital, School of Medicine, Shanghai Jiao Tong University. Written informed consent was obtained from each subject before participation. All procedures were in accordance with the institutional guidelines. 
TABLE 1 | Neuropsychological tests used to evaluate cognitive status.

\begin{tabular}{ll}
\hline $\begin{array}{l}\text { Attention/executive } \\
\text { function }\end{array}$ & $\begin{array}{l}\text { Chinese modified version of the Trail Making Test (TMT) } \\
\text { Modified version of the Stroop Color-Word Test (SCWT) } \\
\text { Category Verbal Fluency Test (VFT) } \\
\text { Memory }\end{array}$ \\
& $\begin{array}{l}\text { Chinese version of the Auditory Verbal Learning Test } \\
\text { (AVLT) for short-delay and long-delay free recall } \\
\text { Rey-Osterrieth Complex Figure (ROCF) delayed recall } \\
\text { test (Chinese version) }\end{array}$ \\
& Boston Naming Test (the 30-item version) \\
Language & ROCF copy test
\end{tabular}

\section{Neuropsychological Assessment}

Neuropsychological assessments were performed by two experienced neurologists (QX and WC) within 1 week of the MRI examination. No patients suffered any transient ischemic attacks or strokes between the MRI examination and the assessment. A comprehensive battery of neuropsychological tests was designed to evaluate cognitive status, which included all cognitive domains. The scales listed in Table $\mathbf{1}$ were used as described in previous studies $(40,41)$. To assess the cognitive status of each patient, the norms used were based on the mean score of each measurement, which were derived from a smallscale normative study of a community of healthy elderly people in Shanghai, China (42). Cognitive dysfunction was defined as $-1.5 \mathrm{SD}$ on at least one neuropsychological test.

To allow direct comparisons among different neuropsychological tests, a z-score was calculated for each neuropsychological test. A $\mathrm{z}$-score is defined as a score that falls in the distribution of normal scores. A $\mathrm{z}$-score of +1.0 corresponds to a score $1 \mathrm{SD}$ above the mean score. The raw scores for each neuropsychological measure were $\mathrm{z}$-transformed. Then, the $z$-scores for each domain were generated by averaging the $z$-scores of their respective tests. Composite z-scores of global cognition, which represented general intellectual ability, were computed by averaging the z-scores of all four cognitive domains.

\section{MRI Acquisition}

All MRI data were obtained using a 3.0T MRI scanner (Signa HDxt; GE HealthCare, Milwaukee, WI, USA) equipped with an eight-channel phased array head coil at Ren Ji Hospital. Each subject underwent three dimensional T1 high-resolution imaging and FLAIR scans. All subjects underwent MR diffusion tensor imaging (DTI) scans. Fiftysix of the seventy three subjects underwent three-dimensional arterial spin labeling (3DASL) scans. The parameters of each sequence were as follows: (1) sagittal 3D T1 highresolution imaging [repetition time $(\mathrm{TR})=5.6 \mathrm{~ms}$, echo time $(\mathrm{TE})=1.8 \mathrm{~ms}$, inversion time $(\mathrm{TI})=450 \mathrm{~ms}$, flip angle $=15^{\circ}$, slice thickness $=1.0 \mathrm{~mm}$, number of slices $=156$, gap $=0$, field of view $(\mathrm{FOV})=256 \mathrm{~mm} \times 256 \mathrm{~mm}$, and matrix $=256 \times 256] ;(2)$ axial FLAIR $(\mathrm{TR}=9,075 \mathrm{~ms}$, $\mathrm{TE}=150 \mathrm{~ms}, \mathrm{TI}=2,250 \mathrm{~ms}, \mathrm{FOV}=256 \mathrm{~mm} \times 256 \mathrm{~mm}$, matrix $=256 \times 256$, slice thickness $=2 \mathrm{~mm}$, and number of slices $=66$ ); (3) 3D ASL perfusion images were acquired using 3D fast spin-echo acquisition with background suppression and with a labeling duration of $1,500 \mathrm{~ms}$ and a post labeling delay of $2,000 \mathrm{~ms}$. ( $\mathrm{TR}=4,337 \mathrm{~ms}, \mathrm{TE}=9.8 \mathrm{~ms}$, FOV $=240 \mathrm{~mm} \times 240 \mathrm{~mm}$, slice thickness $=4 \mathrm{~mm}$, flip angle $=155^{\circ}, \mathrm{NEX}=3$, and number of slices $=34$ ); (4) DTI $(\mathrm{TR}=17,000 \mathrm{~ms}, \mathrm{TE}=89.8 \mathrm{~ms}$, slice thickness $=2 \mathrm{~mm}$, gap $=0, F O V=256 \mathrm{~mm} \times 256 \mathrm{~mm}$, number of slices $=66$, matrix $=128 \times 128$, and 20 diffusion-weighted directions with b value $=1,000 \mathrm{~s} / \mathrm{mm}^{2}$ ).

\section{Image Processing and Analysis}

Processing of the diffusion MRI dataset was implemented using a pipeline toolbox, PANDA v1.3.1(https://www.nitrc.org/ projects/panda), which is based on FSL tools (43). In the pipeline, skull-stripping with the brain extraction tool (BET) was done to extract brain tissue for b0 image in each subject. Eddy current-induced distortion and head motion artifacts were corrected by registering each raw diffusion-weighted image to the b0 image with an affine transformation. Then diffusion metrics, including $\mathrm{FA}$ and $\mathrm{MD}$, were calculated within a mask created from b0 image. To derive the CBF map of each subject, the three runs of ASL images were inspected, and data with excessive head movement $\left(\geq 2 \mathrm{~mm}\right.$ or $2^{\circ}$ ) were discarded, and the three runs were concatenated. Quantitative CBF was then calculated on a voxel basis according to Wang et al. (44). Voxel-wise partial volume correction was performed (45). Then, the CBF, FA and MD maps for each subject were coregistered to the corresponding individual 3D T1-weighted images using SPM8 (http://www.fil.ion.ucl.ac.uk/spm/software/spm8/).

WMHs were segmented by the lesion growth algorithm as implemented in the LST toolbox version 1.1.4 (www.statistical-modeling.de/lst.html) for SPM (46). Then, WMH clusters were separated into PVWMHs and DWMHs according to the "continuity to ventricle rule" (47). PVWMHs were defined as WMHs that were continuous with the margin of the lateral ventricle and all others were defined as DWMHs. Finally, probabilistic maps for WMH and WM were processed with binarization.

To assess the WMH penumbra for each imaging measure, a NAWM layer mask for each individual dataset was created by linearly aligning the defined binary WMHs to the T1weighted images, according to previous studies $(27,28)$. The NAWM layer mask consisted of 15 layers of PVWMH and DWMH separately. Each layer was parallel and gradually dilated away from the WMH by $1 \mathrm{~mm}$. The innermost layer, closest to the WMH, was defined as layer 1 (NAWM-L1), and the outermost layer was layer 15 (NAWM-L15). To prevent overlapping between layers of neighboring WMHs, the WMH and previous NAWM layers were merged together to create a new "WMH" before creating the next layer. To reduce the partial volume effects of the gray matter (GM) and cerebrospinal fluid (CSF), the GM and CSF maps were dilated by 2 voxels, and subtracted from the NAWM layers. The spatial relationship between WMH and NAWM layer mask is shown in Figure 1. 


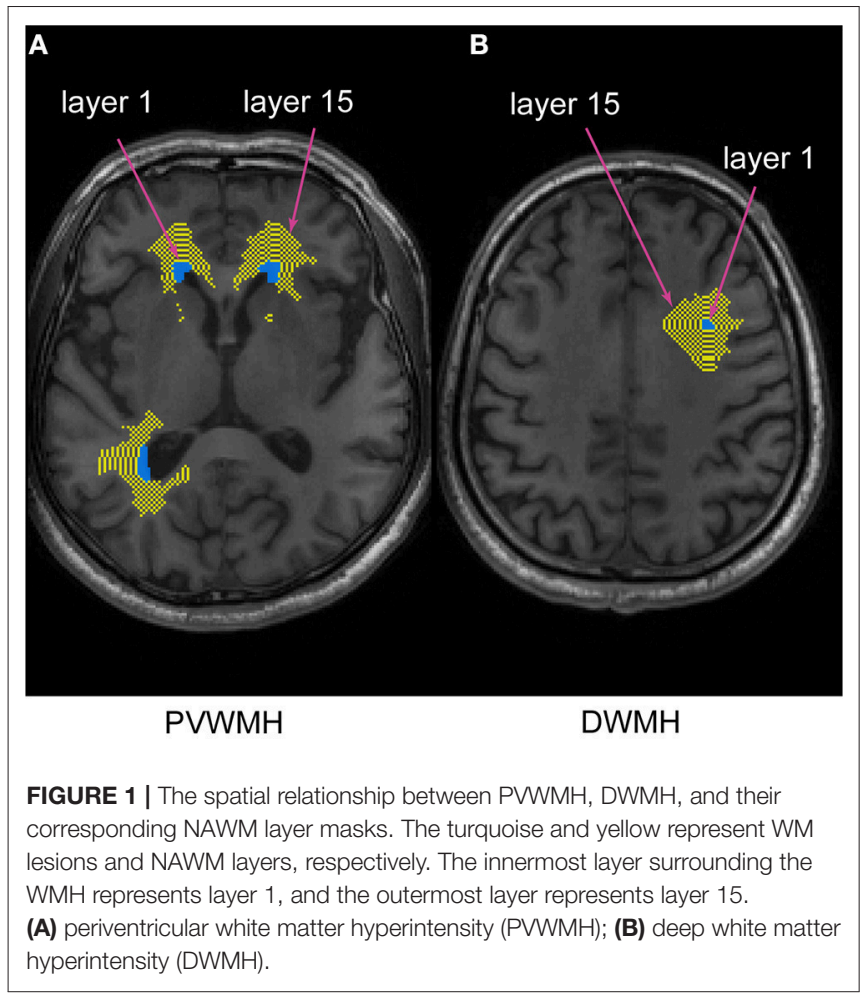

For each subject, the NAWM layer mask was applied to the $\mathrm{FA}, \mathrm{MD}$, and $\mathrm{CBF}$ maps which were previously coregistered to individual 3D T1-weighted images. Later, the mean FA, MD, and CBF values of each NAWM layer for the PVWMH and the DWMH were computed. Similarly, the imaging parameter values of whole-brain WMH and its subtype and whole-brain NAWM were obtained.

\section{Statistical Analysis}

The analyses were performed using IBM SPSS Statistics 20 and $\mathrm{R}$ version 3.5.0. To determine the extent of $\mathrm{WMH} \mathrm{CBF}$ penumbra, mean CBF of the WMH and NAWM layers (L1L15) were compared with the corresponding mean CBF of whole-brain NAWM using a paired $t$-test. The first layer whose CBF value was not significantly different from the whole-brain NAWM was defined as the outer boundary of the CBF penumbra. Due to the impact of WM association and commissural fibers surrounding the ventricles on DTI parameters, the extent of WMH structural penumbra was determined by comparing every two adjacent NAWM layers using paired $t$-tests. The first of two neighboring layers whose values were not significantly different from each other was defined as the outer boundary of the structural penumbra. The PVWMH and DWMH were analyzed separately. Pearson's partial correlations were used to assess the relation between the mean CBF, FA, and MD values of PVWMH, DWMH, and their corresponding penumbras with composite $\mathrm{z}$-scores, controlling for age, sex, and education. A significant difference was set at $p \leq 0.05$.
TABLE 2 | Summary of participant characteristics.

\begin{tabular}{lc}
\hline Variables & Mean \pm SD (range) \\
\hline Number of subjects & 73 \\
Age (years) & $65.71 \pm 8.2(\sim 50-86)$ \\
Female & 25 \\
Years of education & $10.47 \pm 3.07(\sim 6-18)$ \\
Z-scores of attention/executive function & $-1.45 \pm 1.26(\sim-5.33-1.12)$ \\
Z-scores of memory & $-1.46 \pm 0.83(\sim-3.05-0.48)$ \\
Z-scores of language & $-0.75 \pm 1.56(\sim-5.05-1.79)$ \\
Z-scores of visuospatial function & $0.22 \pm 1.99(\sim-8.19-1.46)$ \\
Composite -scores of global cognition & $-0.86 \pm 0.87(\sim-4.04-0.66)$
\end{tabular}

SD, standard deviation.

\section{RESULTS}

\section{Participant Characteristics}

The demographic and cognitive characteristics were presented in Table 2. Seventy-three subjects were included in our study. Their age ranged from 50 to 86 years, with the mean years of education $10.47 \pm 3.07$. The composite z-scores of global cognition were $-0.86 \pm 0.87$, and the $\mathrm{z}$-scores of four cognitive domain (attention/executive function, memory, language and visuospatial function) were $-1.45 \pm 1.26,1.46 \pm 0.83,-0.75 \pm 1.56$ and $0.22 \pm 1.99$, respectively.

\section{The Mean FA, MD, and CBF Values of Each NAWM Layer of the PVWMH and DWMH Are Presented in Table 3 and Figure 2}

The extents of the PVWMH penumbras are as follows: $10 \mathrm{~mm}$ for the CBF penumbra; $6 \mathrm{~mm}$ for the FA penumbra; $6 \mathrm{~mm}$ for the MD penumbra. The extents of the DWMH penumbras are as follows: $7 \mathrm{~mm}$ for the $\mathrm{CBF}$ penumbra; $4 \mathrm{~mm}$ for the $\mathrm{FA}$ penumbra; $2 \mathrm{~mm}$ for the MD penumbra (see Tables S1-S3).

\section{Relations Between the Mean FA, MD, and CBF Values of the PVWMH, DWMH, and Their Penumbras With Composite Z-Scores of Global Cognition and the z-Scores of Each Cognitive Domain Are Illustrated in Table 4 and Tables S4-S7}

Only the mean FA value of the PVWMH-FA penumbra was correlated with the composite $\mathrm{z}$-scores of global cognition ( $r=0.268, p=0.024)$ before correction, as showed in Figure 3. After false discovery rate correction, that correlation did not survive.

\section{DISCUSSION}

For SVCI subjects and cognitively intact elder individuals, the morphological characteristics and distribution features of WM lesions have been widely reported to be associated with cognitive decline $(12,13,48,49)$, whereas few studies have been conducted to explore the association between $\mathrm{WMH}$ penumbras and 
TABLE 3 | The mean FA, MD, and CBF values of the PWWMH, DWMH, and their corresponding layers (mean \pm SD).

\begin{tabular}{|c|c|c|c|c|c|c|}
\hline & \multicolumn{2}{|c|}{ CBF(ml/100 g-tissue/min) } & \multicolumn{2}{|c|}{ FA } & \multicolumn{2}{|c|}{$\mathrm{MD}\left(10^{-4} \mathrm{~mm}^{2} / \mathrm{s}\right)$} \\
\hline & PVWMH & DWMH & PVWMH & DWMH & PVWMH & DWMH \\
\hline WMH & $22.20 \pm 6.85$ & $30.72 \pm 9.74$ & $0.266 \pm 0.031$ & $0.289 \pm 0.057$ & $13.62 \pm 1.52$ & $10.34 \pm 1.77$ \\
\hline Layer 1 & $23.66 \pm 7.05$ & $29.94 \pm 8.83$ & $0.310 \pm 0.041$ & $0.310 \pm 0.056$ & $11.48 \pm 1.46$ & $9.86 \pm 1.60$ \\
\hline Layer 2 & $24.73 \pm 7.11$ & $30.58 \pm 8.67$ & $0.322 \pm 0.048$ & $0.316 \pm 0.056$ & $10.99 \pm 1.56$ & $9.69 \pm 1.53$ \\
\hline Layer 3 & $25.85 \pm 7.22$ & $30.93 \pm 8.62$ & $0.329 \pm 0.052$ & $0.321 \pm 0.056$ & $10.75 \pm 1.52$ & $9.68 \pm 1.59$ \\
\hline Layer 4 & $26.97 \pm 7.29$ & $31.31 \pm 8.67$ & $0.334 \pm 0.053$ & $0.324 \pm 0.054$ & $10.51 \pm 1.46$ & $9.63 \pm 1.63$ \\
\hline Layer 5 & $28.06 \pm 7.39$ & $31.68 \pm 8.76$ & $0.337 \pm 0.055$ & $0.325 \pm 0.052$ & $10.37 \pm 1.50$ & $9.75 \pm 1.67$ \\
\hline Layer 6 & $29.11 \pm 7.53$ & $32.18 \pm 8.91$ & $0.339 \pm 0.056$ & $0.325 \pm 0.050$ & $10.18 \pm 1.43$ & $9.72 \pm 1.67$ \\
\hline Layer 7 & $30.02 \pm 7.62$ & $32.72 \pm 9.05$ & $0.338 \pm 0.056$ & $0.325 \pm 0.048$ & $10.11 \pm 1.36$ & $9.77 \pm 1.74$ \\
\hline Layer 8 & $31.22 \pm 7.72$ & $33.18 \pm 9.14$ & $0.338 \pm 0.056$ & $0.325 \pm 0.048$ & $10.03 \pm 1.33$ & $9.75 \pm 1.69$ \\
\hline Layer 9 & $32.25 \pm 7.85$ & $33.72 \pm 9.26$ & $0.336 \pm 0.055$ & $0.325 \pm 0.046$ & $10.03 \pm 1.30$ & $9.73 \pm 1.72$ \\
\hline Layer 10 & $33.17 \pm 7.97$ & $34.10 \pm 9.28$ & $0.332 \pm 0.055$ & $0.324 \pm 0.045$ & $10.01 \pm 1.29$ & $9.75 \pm 1.73$ \\
\hline Layer 11 & $33.82 \pm 8.37$ & $34.40 \pm 9.19$ & $0.328 \pm 0.055$ & $0.323 \pm 0.044$ & $10.00 \pm 1.26$ & $9.83 \pm 1.69$ \\
\hline Layer 12 & $34.67 \pm 8.13$ & $34.88 \pm 9.20$ & $0.324 \pm 0.054$ & $0.321 \pm 0.043$ & $9.99 \pm 1.27$ & $9.83 \pm 1.60$ \\
\hline Layer 13 & $35.28 \pm 8.14$ & $35.26 \pm 9.24$ & $0.320 \pm 0.054$ & $0.319 \pm 0.042$ & $9.96 \pm 1.31$ & $9.92 \pm 1.61$ \\
\hline Layer 14 & $35.77 \pm 8.18$ & $35.52 \pm 9.18$ & $0.315 \pm 0.054$ & $0.317 \pm 0.042$ & $9.97 \pm 1.32$ & $9.94 \pm 1.60$ \\
\hline Layer 15 & $36.21 \pm 8.30$ & $35.76 \pm 8.93$ & $0.311 \pm 0.056$ & $0.315 \pm 0.042$ & $10.01 \pm 1.37$ & $9.97 \pm 1.53$ \\
\hline WBNAWM & $33.45 \pm 8.58$ & $33.45 \pm 8.58$ & $0.312 \pm 0.026$ & $0.312 \pm 0.026$ & $9.19 \pm 0.74$ & $9.19 \pm 0.74$ \\
\hline
\end{tabular}

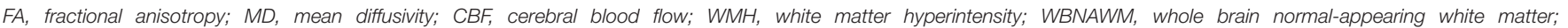
$S D$, standard deviation.

cognitive function and the contribution of subtle abnormalities within the penumbras to cognitive function remains unclear. The present study aimed to explore the role of $\mathrm{WMH}$ penumbras in global cognition of svMCI subjects. Our results revealed reduced $\mathrm{CBF}$ and $\mathrm{FA}$, and increased $\mathrm{MD}$, of $\mathrm{WMH}$ and its surrounding NAWM for both PVWMH and DWMH. Our findings also showed that the CBF penumbra was wider than the structural penumbra as defined by FA and MD.

Our finding that CBF penumbras were more extensive than structural penumbras around both PVWMH and DWMH was consistent with a previous study (27). Wider CBF penumbras covered structural penumbras and no microstructural changed NAWM, suggesting the likelihood that compromised CBF precedes white matter integrity changes. Longitudinal research is needed to determine whether perfusion or structural changes come first. CBF plays a critical role in the maintenance of neuronal integrity, and the $\mathrm{CBF}$ penumbra may reflect more extensive white matter alterations beyond the structural penumbra. A previous longitudinal study found that NAWM voxels that converted to new WMH at follow-up had significantly lower baseline CBF than persistent NAWM voxels that did not convert to $\mathrm{WMH}$, suggesting that the CBF penumbra is linked to WMH extension (28). Lower CBF within NAWM reflects a higher risk for future conversion to WMH. Thus, the CBF penumbras of both PVWMH and DWMH can be considered novel targets for prediction and intervention of WMH progression. Our findings showed that CBF gradually increased from NAMW layer 1 to layer 15, suggesting that subtle NAWM injury is likely a continuous process, and WMH growth is mostly from inner NAWM layers of existing WMHs to outer layers. This finding supported those of previous studies, revealing that most growing WMHs extended from the edge of existing lesions to the outer region $(22,28)$.

Consistent with previous studies, we found that the FA and MD penumbras covered 2-6 mm from WMHs (22, 27, 29). Our results showed that the FA values of the inner periventricular NAWM layers were slightly higher than those of the outer periventricular NAWM layers and the mean whole-brain NAWM. The "location effect" may explain this phenomenon (27). PVWMHs are located near highly organized myelinated structures, such as the corpus callosum, where water diffusion is highly restricted and dependent on fiber direction. Other studies have also shown similar phenomena $(27,50)$. DTI measures of WM microstructural integrity appeared to provide an earlier indication of WM injury than WMHs. A previous study demonstrated that NAWM regions converting to WMH had significantly lower FA and higher MD values than persistent NAWM regions, suggesting that changes in NAWM precede the development of WM lesions (33). Moreover, vast number of studies have revealed that the pathophysiological alterations in WM is a gradual process and WMHs are only the tip of the iceberg of WM pathology (23, 29, 51, 52). Decreased FA and increased MD within NAWM and WMH indicate microstructural WM integrity disruption and altered water mobility, which may affect the integrity of WM tracts connecting cortical-subcortical areas, leading to disconnection syndrome and cognitive decline $(53,54)$.

Only the PVWMH-FA penumbra was correlated with global cognition, whereas that correlation did not survive after correcting the $p$-value for multiple comparisons. The relationship 
PWWMH

A

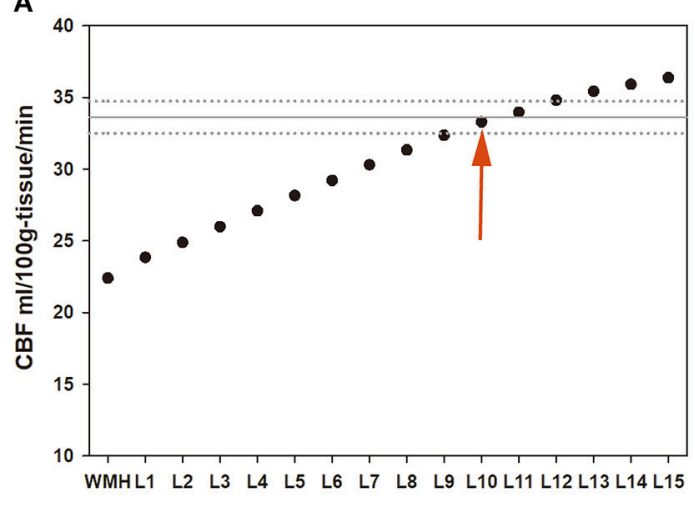

B

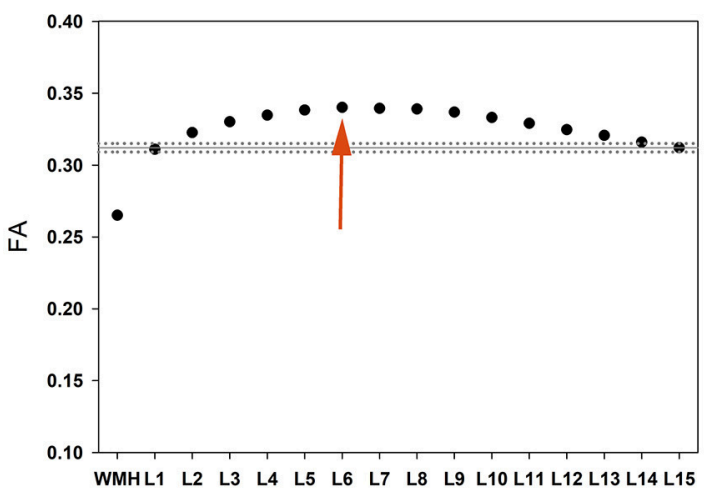

C

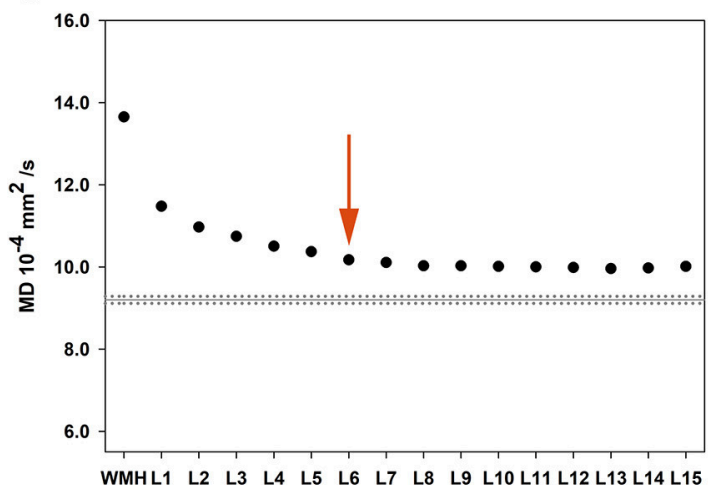

DWMH

D
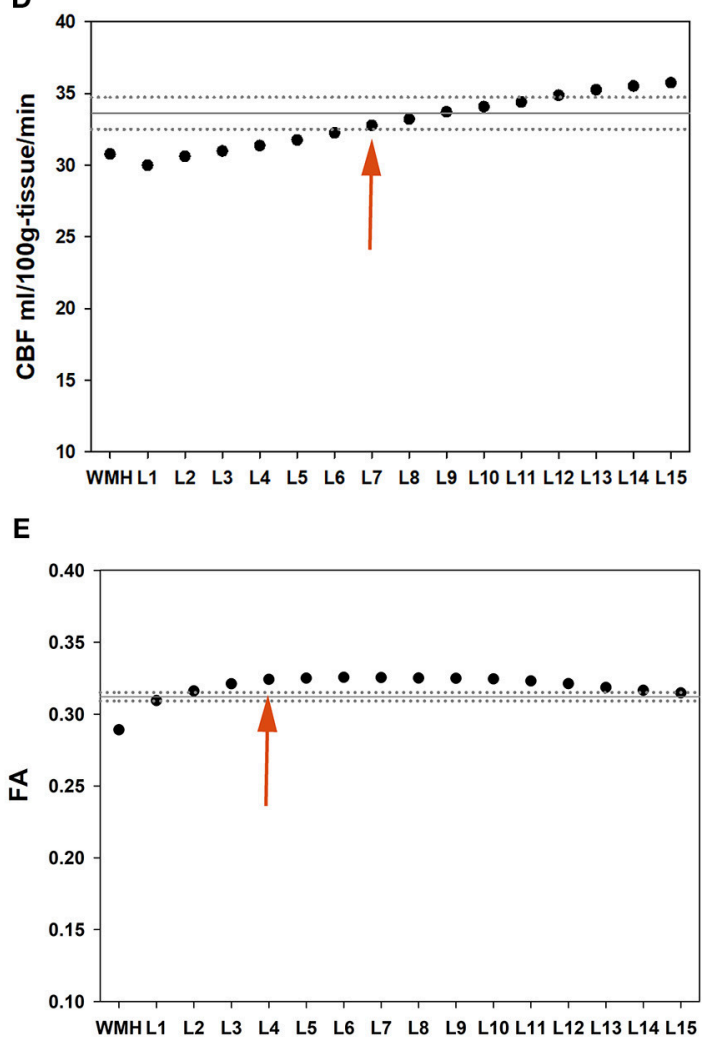

F

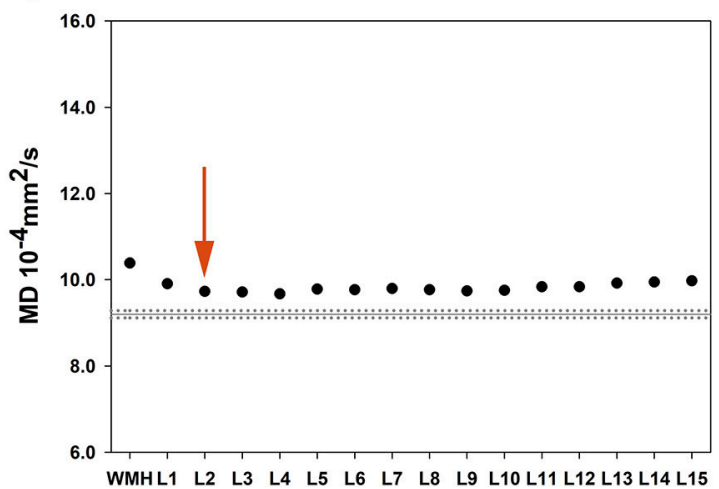

FIGURE 2 | Group means of the PWWMH (the left column) and DWMH (the right column) and their outer NAWM layers. The solid horizontal and dotted lines represent the mean and standard error of the whole-brain NAWM CBF, FA, and MD values, respectively. Red arrows represent the outer boundary of WMH penumbra for each dataset. (A) PWWMH-CBF; (B) PWWMH-FA; (C) PWWMH-MD; (D) DWMH-CBF; (E) DWMH-FA; (F) DWMH-MD.

between penumbra findings and cognition was weak. This likely represents the fact that these physiological processes of penumbra are no destructive per se but are established risk factors for future brain injury that will associated with progressive cognitive impairment. However, the exact reason underlying this occurrence is not well-established, and further studies are needed.

Our finding that there was no significant correlation between the imaging parameters within WMHs, including the two classifications, and global cognitive function was not consistent with those of previous studies $(55,56)$. A previous study revealed that global cognitive function, assessed by Mini-Mental State Examination (MMSE), was associated with total brain NAWMFA and WMH-FA in subjects with leukoaraiosis using a multiple linear regression analysis (55). A population-based study also showed that MD, radial diffusivity and axial diffusivity of both $\mathrm{WMH}$ and NAWM were associated with global cognition, 
TABLE 4 | Relations between the mean FA, MD, and CBF values of PWWMH, DWMH, and their penumbras with composite z-scores of global cognition.

\begin{tabular}{|c|c|c|c|c|}
\hline & $\begin{array}{c}\text { Mean values of imaging } \\
\text { parameters }\end{array}$ & $r$ & $p$-value & $\begin{array}{c}p \text {-value corrected by } \\
\text { FDR }\end{array}$ \\
\hline PWWMH-CBF & 22.20 & 0.112 & 0.421 & 0.6495 \\
\hline PWWMH-FA & 0.267 & 0.115 & 0.341 & 0.6495 \\
\hline PWWMH-MD & 13.62 & 0.077 & 0.522 & 0.6960 \\
\hline DWMH-CBF & 30.72 & 0.171 & 0.226 & 0.6945 \\
\hline DWMH-FA & 0.289 & 0.046 & 0.707 & 0.7370 \\
\hline DWMH-MD & 10.34 & -0.13 & 0.286 & 0.6945 \\
\hline Mean CBF of PWWMH-CBF penumbra & 28.52 & -0.047 & 0.737 & 0.7370 \\
\hline Mean FA of PWWMH-FA penumbra & 0.328 & 0.268 & 0.024 & 0.2880 \\
\hline Mean MD of PWWMH-MD penumbra & 10.71 & -0.047 & 0.696 & 0.7370 \\
\hline Mean CBF of DWMH-CBF penumbra & 31.33 & 0.213 & 0.129 & 0.6945 \\
\hline Mean FA of DWMH-FA penumbra & 0.318 & 0.096 & 0.433 & 0.6945 \\
\hline Mean MD of DWMH-MD penumbra & 9.8 & -0.110 & 0.370 & 0.6945 \\
\hline
\end{tabular}

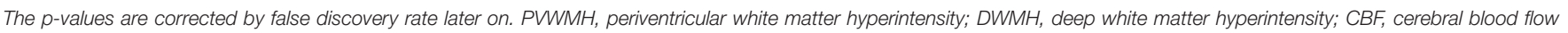
(m//100 g-tissue/min); FA, fractional anisotropy; $M D$, mean diffusivity $\left(10^{-4} \mathrm{~mm}^{2} / \mathrm{s}\right) ; \mathrm{FDR}$, false discovery rate.

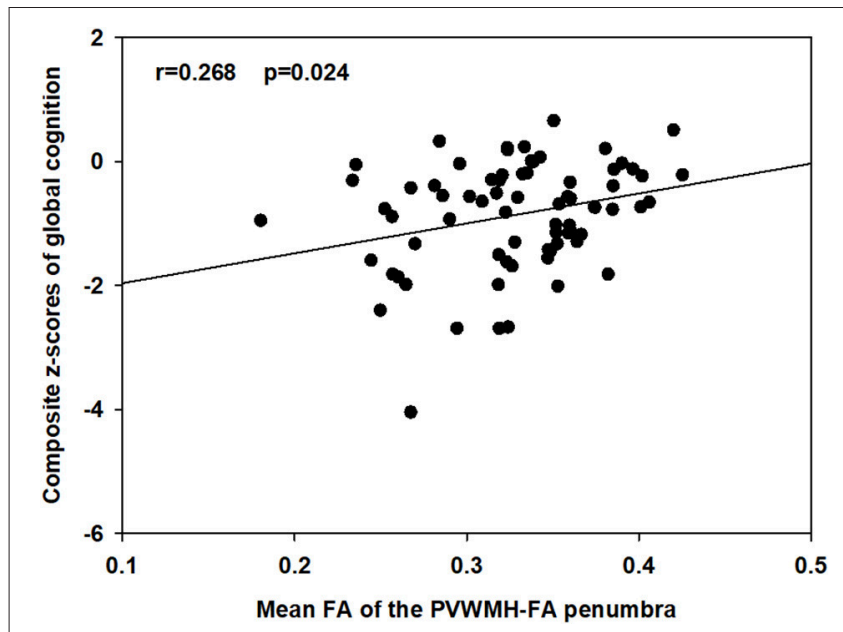

FIGURE 3 | Associations between the mean FA of the PVWMH-FA penumbra and the composite z-scores of global cognition. Partial $r$ and $p$-values was obtained after controlling for age, sex, and education.

regardless of white matter atrophy and WMH volume (57). The lack of correlation between imaging parameters and cognition in our study, which was inconsistent with these previous studies, may be attributed to two aspects as follows. On the one hand, this discrepancy may be attributed to the different participants and methods used to assess cognitive status. On the other hand, the heterogeneity of WMHs may partly explain this discrepancy. Postmortem studies have revealed that WMHs are histopathologically heterogeneous in both severity and nature (26). The spatial distribution and the signal properties, including magnetization transfer imaging, DTI and FLAIR, of WMHs have also been revealed to be heterogeneous, though WMHs have similar appearances on FLAIR and T2-weighted images $(32,58,59)$. Given that WMHs are heterogeneous in terms of histopathology, spatial distribution and the signal properties, mixed analysis of WMHs may compromise the reliability of the analysis in assessing the association of WMHs with cognitive function, which may partly explain our finding. Though it is not feasible or realistic to control all the heterogeneities of WMHs in one study, future studies should be conducted to stratify WMHs according to their heterogeneities.

Measures of microstructural integrity and perfusion within WMH penumbra may have several clinical implications. First, to prevent WMH growth, it is crucial to understand the etiology of development of NAWM tissue within WMH penumbra into WMH which represents more severe WM injury. A longitudinal study had showed that radial diffusivity, reflecting demyelination $(60,61)$, had the strongest relationship with WMH expansion compared to axial diffusivity and CBF, indicating that demyelination may be the main underlying etiology of WMH development (62). Second, previous longitudinal studies had found that some WMH may regress after minor stroke, with potentially better clinical and brain tissue outcomes, suggesting that WMH reversibility may attribute to the transient disturbance of the blood-brain barrier (BBB), causing interstitial fluid alterations (63-66). A longitudinal study revealed that participants with WMH decrease had larger reductions in blood pressure and $\mathrm{MD}$ in NAWM than participants with $\mathrm{WMH}$ increase (66). Thus, ASL CBF and DTI measures may be used to investigate cerebral perfusion and microstructural integrity as the potential mechanism explaining the reversal of $\mathrm{WMH}$ and $\mathrm{WMH}$ penumbra. Third, a clinician should take into account that the true WM injury may be more extensive than the visible WMH. WMH penumbra represents milder WM injury concerning $\mathrm{WMH}$ and may be a high-priority target for intervention as it is potentially reversible to treatment.

There were several limitations to our study. First, because there is no generally adopted rule for defining the extent of WMH penumbras to date, we defined the $\mathrm{CBF}$ penumbra by comparing the mean $\mathrm{CBF}$ of each NAWM layer to that of the whole-brain NAWM. Whole-brain NAWM already contained subtle changes in the NAWM. Thus, the CBF penumbra defined by this method 
is slightly narrower than the real CBF penumbra. Second, the number of lacunes, WMH location and corresponding cortical dysfunction also have an impact on cognitive impairments (67, 68). The associations between WMHs and their penumbras with cognition may be mediated by these confounders. In the present study, we only investigated the effect of WMH penumbras on cognitive status. Our future work will seek to quantify and model these confounders for the purpose of better understanding the role of WMH penumbra in cognition. Third, in this study, we assumed that WMHs equably affects surrounding NAMW along the straight line between the two, without taking the architecture of WM fibers into consideration. It may be more biologically plausible to assume that a WMH at one location on a WM fiber more strongly influences WM integrity along the rest of the same tract than in other tracts. However, it seems to be unfeasible and unrealistic to use DTI tractography to investigate each WMH and its penumbra one by one. Finally, our study was a cross-sectional study, and the sample size was small. Longitudinal studies with larger sample sizes are needed.

\section{CONCLUSION}

In this study, reduced $\mathrm{CBF}$ and $\mathrm{FA}$ and increased $\mathrm{MD}$ in the inner NAWM layers for both PVWMH and DWMH suggested extensive WM alterations beyond the visible WM lesions commonly observed on clinical MRI of svMCI subjects. $\mathrm{CBF}$ penumbras cover more extensive WM at risk than DTI penumbras, suggesting the likelihood that compromised $\mathrm{CBF}$ precedes white matter integrity changes, and $\mathrm{CBF}$ penumbras may be a potential target for the prevention of further microstructural white matter damage. The imaging parameters investigated, however, did not correlate to cognition.

\section{REFERENCES}

1. O'Brien JT, Erkinjuntti T, Reisberg B, Roman G, Sawada T, Pantoni L, et al. Vascular cognitive impairment. Lancet Neurol. (2003) 2:89-98. doi: 10.1016/S1474-4422(03)00305-3

2. Roh JH, Lee JH. Recent updates on subcortical ischemic vascular dementia. $J$ Stroke. (2014) 16:18-26. doi: 10.5853/jos.2014.16.1.18

3. Lee MJ, Seo SW, Na DL, Kim C, Park JH, Kim GH, et al. Synergistic effects of ischemia and beta-amyloid burden on cognitive decline in patients with subcortical vascular mild cognitive impairment. JAMA Psychiatry. (2014) 71:412-22. doi: 10.1001/jamapsychiatry.2013.4506

4. Wardlaw JM, Smith C, Dichgans M. Mechanisms of sporadic cerebral small vessel disease: insights from neuroimaging. Lancet Neurol. (2013) 12:483-97. doi: 10.1016/S1474-4422(13)70060-7

5. Moran C, Phan TG, Srikanth VK. Cerebral small vessel disease: a review of clinical, radiological, and histopathological phenotypes. Int J Stroke. (2012) 7:36-46. doi: 10.1111/j.1747-4949.2011.00725.x

6. de Leeuw FE, de Groot JC, Oudkerk M, Kors JA, Hofman A, van Gijn J, et al. Atrial fibrillation and the risk of cerebral white matter lesions. Neurology. (2000) 54:1795-801. doi: 10.1212/WNL.54.9.1795

7. Vermeer SE, van Dijk EJ, Koudstaal PJ, Oudkerk M, Hofman A, Clarke R, et al. Homocysteine, silent brain infarcts, and white matter lesions: the Rotterdam Scan Study. Ann Neurol. (2002) 51:285-9. doi: 10.1002/ana.10111

8. de Leeuw FE, de Groot JC, Oudkerk M, Witteman JC, Hofman A, van Gijn J, et al. Hypertension and cerebral white matter lesions in a prospective cohort study. Brain. (2002) 125:765-72. doi: 10.1093/brain/awf077

\section{AUTHOR CONTRIBUTIONS}

YZ, QX, and XG conceived and designed the experiments. XW, $\mathrm{XH}, \mathrm{JD}, \mathrm{YS}, \mathrm{WD}$, and MC performed the experiments. XW, XG, and YW analyzed the data. XW, XG, and JD contributed reagents, materials, analysis tools. XW and YW wrote the paper. XW and JD figures processing.

\section{FUNDING}

This work was supported by the Shanghai Science and Technology Committee Medical Guide Project (western medicine; No. 17411964300), and the Shanghai Municipal Education Commission-Gaofeng Clinical Medicine Grant Support (No. 20172013), the National Natural Science Foundation of China (No. 81571650), the Medical Engineering Cross Research Foundation of Shanghai JiaoTong University (No. YG2017QN47), and the Research Seed Fund of Ren Ji Hospital, School of Medicine, Shanghai Jiao Tong University (RJZZ18-003), the national Key Research and Development Program of China 2016YFC1300600, and the Incubating Program for Clinical Research and Innovation of Ren Ji Hospital, School of Medicine, Shanghai Jiao Tong University (PYIV-17-003 and PYIII-17-027). The funders had no role in study design, data collection and analysis, decision to publish, or preparation of the manuscript.

\section{SUPPLEMENTARY MATERIAL}

The Supplementary Material for this article can be found online at: https://www.frontiersin.org/articles/10.3389/fneur. 2019.00348/full\#supplementary-material

9. Vermeer SE, Hollander M, van Dijk EJ, Hofman A, Koudstaal PJ, Breteler $\mathrm{MM}$, et al. Silent brain infarcts and white matter lesions increase stroke risk in the general population: the Rotterdam Scan Study. Stroke. (2003) 34:1126-9. doi: 10.1161/01.STR.0000068408.82115.D2

10. de Groot JC, de Leeuw FE, Oudkerk M, Hofman A, Jolles J, Breteler MM. Cerebral white matter lesions and subjective cognitive dysfunction: the Rotterdam Scan Study. Neurology. (2001) 56:1539-45. doi: 10.1212/WNL.56.11.1539

11. Ylikoski R, Ylikoski A, Erkinjuntti T, Sulkava R, Raininko R, Tilvis R. White matter changes in healthy elderly persons correlate with attention and speed of mental processing. Arch Neurol. (1993) 50:818-24. doi: 10.1001/archneur.1993.00540080029009

12. Bolandzadeh N, Davis JC, Tam R, Handy TC, Liu-Ambrose T. The association between cognitive function and white matter lesion location in older adults: a systematic review. BMC Neurology. (2012) 12:126. doi: 10.1186/1471-2377-12-126

13. Dhamoon MS, Cheung YK, Bagci A, Alperin N, Sacco RL, Elkind MSV, et al. Periventricular white matter hyperintensities and functional decline. J Am Geriatr Soc. (2018) 66:113-9. doi: 10.1111/ jgs. 15149

14. Kim KW, MacFall JR, Payne ME. Classification of white matter lesions on magnetic resonance imaging in elderly persons. Biol Psychiatry. (2008) 64:273-80. doi: 10.1016/j.biopsych.2008.03.024

15. Schmidt R, Schmidt H, Haybaeck J, Loitfelder M, Weis S, Cavalieri M, et al. Heterogeneity in age-related white matter changes. Acta Neuropathol. (2011) 122:171-85. doi: 10.1007/s00401-011-0851-x 
16. Wardlaw JM, Valdés Hernández MC, Muñoz-Maniega S, Wardlaw JM, Valdes Hernandez MC, Munoz-Maniega S. What are white matter hyperintensities made of? Relevance to vascular cognitive impairment. J Am Heart Assoc. (2015) 4:001140. doi: 10.1161/JAHA.114.001140

17. Fazekas F, Schmidt R, Scheltens P. Pathophysiologic mechanisms in the development of age-related white matter changes of the brain. Dementia Geriatr Cogn Disord. (1998) 9:2-5. doi: 10.1159/000051182

18. Prins ND, Scheltens P. White matter hyperintensities, cognitive impairment and dementia: an update. Nat Rev Neurol. (2015) 11:157-65. doi: 10.1038/nrneurol.2015.10

19. Ding D, Xiong Y, Zhao Q, Guo Q, Chu S, Chu WWC, et al. White matter hyperintensity predicts the risk of incident cognitive decline in community dwelling elderly. J Alzheimer's Dis. (2018) 61:1333-41. doi: 10.3233/JAD-170876

20. Debette S, Markus HS. The clinical importance of white matter hyperintensities on brain magnetic resonance imaging: systematic review and meta-analysis. BMJ. (2010) 341:c3666. doi: 10.1136/bmj.c3666

21. Prins ND, van Dijk EJ, den Heijer T, Vermeer SE, Jolles J, Koudstaal PJ, et al. Cerebral small-vessel disease and decline in information processing speed, executive function and memory. Brain. (2005) 128:2034-41. doi: 10.1093/brain/awh553

22. Maillard P, Carmichael O, Fletcher E, Reed B, Mungas D, DeCarli C. Coevolution of white matter hyperintensities and cognition in the elderly. Neurology. (2012) 79:442-8. doi: 10.1212/WNL.0b013e3182617136

23. Simpson JE, Ince PG, Higham CE, Gelsthorpe CH, Fernando MS, Matthews F, et al. Microglial activation in white matter lesions and nonlesional white matter of ageing brains. Neuropathol Appl Neurobiol. (2007) 33:670-83. doi: 10.1111/j.1365-2990.2007.00890.x

24. van der Valk P, De Groot CJ. Staging of multiple sclerosis (MS). lesions: pathology of the time frame of MS. Neuropathol Appl Neurobiol. (2000) 26:2-10. doi: 10.1046/j.1365-2990.2000.00217.x

25. Gobin SJ, Montagne L, Van Zutphen M, Van Der Valk P, Van Den Elsen PJ, De Groot CJ. Upregulation of transcription factors controlling MHC expression in multiple sclerosis lesions. Glia. (2001) 36:68-77. doi: 10.1002/glia.1096

26. Gouw AA, Seewann A, van der Flier WM, Barkhof F, Rozemuller AM, Scheltens $P$, et al. Heterogeneity of small vessel disease: a systematic review of MRI and histopathology correlations. J Neurol Neurosurg Psychiatry. (2011) 82:126-35. doi: 10.1136/jnnp.2009.204685

27. Promjunyakul NO, Lahna DL, Kaye JA, Dodge HH, Erten-Lyons D, Rooney WD, et al. Comparison of cerebral blood flow and structural penumbras in relation to white matter hyperintensities: a multi-modal magnetic resonance imaging study. J Cereb Blood Flow Metab. (2016) 36:1528-36. doi: $10.1177 / 0271678 X 16651268$

28. Promjunyakul N, Lahna D, Kaye JA, Dodge HH, Erten-Lyons D, Rooney $\mathrm{WD}$, et al. Characterizing the white matter hyperintensity penumbra with cerebral blood flow measures. Neuroimage Clin. (2015) 8:224-9. doi: 10.1016/j.nicl.2015.04.012

29. Maillard P, Fletcher E, Harvey D, Carmichael O, Reed B, Mungas D, et al. White matter hyperintensity penumbra. Stroke. (2011) 42:1917-22. doi: 10.1161/STROKEAHA.110.609768

30. Wang Y, Cao W, Sun Y, Chen X, Ding W, Xu Q, et al. White matter integrity in subcortical vascular cognitive impairment: a multimodal structural MRI Study. Curr Alzheimer Res. (2017) 14:991-9. doi: 10.2174/1567205014666170329113707

31. Zhou Y, Qun-Xu, Qin LD, Qian LJ, Cao WW, Xu JR. A primary study of diffusion tensor imaging-based histogram analysis in vascular cognitive impairment with no dementia. Clin Neurol Neurosurg. (2011) 113:92-7. doi: 10.1016/j.clineuro.2010.09.007

32. Maillard P, Fletcher E, Lockhart SN, Roach AE, Reed B, Mungas $\mathrm{D}$, et al. White matter hyperintensities and their penumbra lie along a continuum of injury in the aging brain. Stroke. (2014) 45:1721-6. doi: 10.1161/STROKEAHA.113.004084

33. de Groot M, Verhaaren BF, de Boer R, Klein S, Hofman A, van der Lugt A, et al. Changes in normal-appearing white matter precede development of white matter lesions. Stroke. (2013) 44:1037-42. doi: 10.1161/STROKEAHA.112.680223

34. Zhang X, Ding L, Yang L, Qin W, Li Y, Li S, et al. Relationship between white matter hyperintensities penumbra and cavity formation. Med Sci Monit. (2016) 22:41-9. doi: 10.12659/ MSM.896324

35. Duering M, Csanadi E, Gesierich B, Jouvent E, Hervé D, Seiler S, et al. Incident lacunes preferentially localize to the edge of white matter hyperintensities: insights into the pathophysiology of cerebral small vessel disease. Brain. (2013) 136:2717-26. doi: 10.1093/brain/awt184

36. Galluzzi S, Sheu CF, Zanetti O, Frisoni GB. Distinctive clinical features of mild cognitive impairment with subcortical cerebrovascular disease. Dement Geriatr Cogn Disord. (2005) 19:196-203. doi: 10.1159/000083499

37. Petersen RC, Smith GE, Waring SC, Ivnik RJ, Tangalos EG, Kokmen E. Mild cognitive impairment: clinical characterization and outcome. Arch Neurol. (1998) 56:303-8. doi: 10.1001/archneur.56.3.303

38. Gorelick PB, Scuteri A, Black SE, Decarli C, Greenberg SM, Iadecola C, et al. Vascular contributions to cognitive impairment and dementia: a statement for healthcare professionals from the american heart association/american stroke association. Stroke. (2011) 42:2672-713. doi: 10.1161/STR.0 b013e3182299496

39. Hamilton M. A rating scale for depression. J Neurol Neurosurg Psychiatry. (1960) 23:56-62. doi: 10.1136/jnnp.23.1.56

40. Hachinski V, Iadecola C, Petersen RC, Breteler MM, Nyenhuis DL, Black SE, et al. National Institute of Neurological Disorders and Stroke-Canadian Stroke Network vascular cognitive impairment harmonization standards. Stroke. (2006) 37:2220-41. doi: 10.1161/01.STR.0000237236.88823.47

41. Xu Q, Cao WW, Mi JH, Yu L, Lin Y, Li YS. Brief screening for mild cognitive impairment in subcortical ischemic vascular disease: a comparison study of the Montreal Cognitive Assessment with the Mini-Mental State Examination. Eur Neurol. (2014) 71:106-14. doi: 10.1159/000353988

42. Guo QH, Sun YM, Yuan J, Hong Z, Lu CZ. Application of eight executive tests in participants at Shanghai communities. Chin J Behav Med. Sci. (2007) 16:628-631. doi: 10.3760/cma.j.issn.1674-6554.2007.07.022

43. Cui Z, Zhong S, Xu P, He Y, Gong G. PANDA: a pipeline toolbox for analyzing brain diffusion images. Front Hum Neurosci. (2013) 7:42. doi: 10.3389/fnhum.2013.00042

44. Wang J, Licht DJ, Jahng GH, Liu CS, Rubin JT, Haselgrove J, et al. Pediatric perfusion imaging using pulsed arterial spin labeling. J Magn Reson Imaging. (2003) 18:404-13. doi: 10.1002/jmri.10372

45. Du AT, Jahng GH, Hayasaka S, Kramer JH, Rosen HJ, Gorno-Tempini ML, et al. Hypoperfusion in frontotemporal dementia and Alzheimer disease by arterial spin labeling MRI. Neurology. (2006) 67:1215-20. doi: 10.1212/01.wnl.0000238163.71349.78

46. Schmidt P, Gaser C, Arsic M, Buck D, Förschler A, Berthele A, et al. An automated tool for detection of FLAIR-hyperintense whitematter lesions in Multiple Sclerosis. Neuroimage. (2012) 59:3774-83. doi: 10.1016/j.neuroimage.2011.11.032

47. Griffanti L, Jenkinson M, Suri S, Zsoldos E, Mahmood A, Filippini N, et al. Classification and characterization of periventricular and deep white matter hyperintensities on MRI: a study in older adults. Neuroimage. (2017) 170:17481. doi: 10.1016/j.neuroimage.2017.03.024

48. Lampe L, Kharabian-Masouleh S, Kynast J, Arelin K, Steele CJ, Löffler $\mathrm{M}$, et al. Lesion location matters: the relationships between white matter hyperintensities on cognition in the healthy elderly. J Cereb Blood Flow Metab. (2017) 39:36-43. doi: 10.1177/0271678X1 7740501

49. de Bresser J, Kuijf HJ, Zaanen K, Viergever MA, Hendrikse J, Biessels GJ, et al. White matter hyperintensity shape and location feature analysis on brain MRI; proof of principle study in patients with diabetes. Sci Rep. (2018) 8:1893. doi: 10.1038/s41598-018-20084-y

50. Maniega SM, Valdés Hernández MC, Clayden JD, Royle NA, Murray C, Morris Z, et al. White matter hyperintensities and normal-appearing white matter integrity in the aging brain. Neurobiol Aging. (2015) 36:909-18. doi: 10.1016/j.neurobiolaging.2014.07.048

51. Gouw AA, van der Flier WM, Fazekas F, van Straaten EC, Pantoni L, Poggesi A, et al. Progression of white matter hyperintensities and incidence of new lacunes over a 3-year period: the Leukoaraiosis and Disability study. Stroke. (2008) 39:1414-20. doi: 10.1161/STROKEAHA.107.498535

52. Ropele S, Seewann A, Gouw AA, van der Flier WM, Schmidt R, Pantoni $\mathrm{L}$, et al. Quantitation of brain tissue changes associated with white matter hyperintensities by diffusion-weighted and magnetization transfer imaging: 
the LADIS (Leukoaraiosis and Disability in the Elderly) study. J Magn Reson Imaging. (2009) 29:268-74. doi: 10.1002/jmri.21580

53. Shenkin SD, Bastin ME, Macgillivray TJ, Deary IJ, Starr JM, Rivers CS, et al. Cognitive correlates of cerebral white matter lesions and water diffusion tensor parameters in community-dwelling older people. Cerebrovasc Dis. (2005) 20:310-8. doi: 10.1159/0000 87930

54. Basser PJ, Pierpaoli C. Microstructural and physiological features of tissues elucidated by quantitative-diffusion-tensor MRI. J Magn Reson. Ser B. (1996) 111:209-19. doi: 10.1006/jmrb.1996.0086

55. Zhong G, Zhang R, Jiaerken Y, Yu X, Zhou Y, Liu C, et al. Better correlation of cognitive function to white matter integrity than to blood supply in subjects with leukoaraiosis. Front Aging Neurosci. (2017) 9:185. doi: 10.3389/fnagi.2017. 00185

56. Chabriat H, Pappata S, Poupon C, Clark CA, Vahedi K, Poupon F, et al. Clinical severity in CADASIL related to ultrastructural damage in white matter: in vivo study with diffusion tensor MRI. Stroke. (1999) 30:2637-43. doi: 10.1161/01.STR.30.12.2637

57. Vernooij MW, Ikram MA, Vrooman HA, Wielopolski PA, Krestin GP, Hofman A, et al. White matter microstructural integrity and cognitive function in a general elderly population. Arch Gen Psychiatry. (2009) 66:54553. doi: 10.1001 /archgenpsychiatry.2009.5

58. Yoshita M, Fletcher E, Harvey D, Ortega M, Martinez O, Mungas $\mathrm{DM}$, et al. Extent and distribution of white matter hyperintensities in normal aging, MCI, and AD. Neurology. (2006) 67:2192-8. doi: 10.1212/01.wnl.0000249119.95747.1f

59. Spilt A, Goekoop R, Westendorp RG, Blauw GJ, de Craen AJ, van Buchem MA. Not all age-related white matter hyperintensities are the same: a magnetization transfer imaging study. Am J Neuroradiol. (2006) 27:1964-8.

60. Schmierer K, Wheeler-Kingshott CA, Tozer DJ, Boulby PA, Parkes HG, Yousry TA, et al. Quantitative magnetic resonance of postmortem multiple sclerosis brain before and after fixation. Magn Reson Med. (2008) 59:268-77. doi: $10.1002 / \mathrm{mrm} .21487$

61. Song SK, Sun SW, Ramsbottom MJ, Chang C, Russell J, Cross AH. Dysmyelination revealed through MRI as increased radial (but unchanged axial) diffusion of water. Neuroimage. (2002) 17:1429-36. doi: 10.1006/nimg.2002.1267
62. Promjunyakul NO, Dodge HH, Lahna D, Boespflug EL, Kaye JA, Rooney WD, et al. Baseline NAWM structural integrity and CBF predict periventricular WMH expansion over time. Neurology. (2018) 90:e2119-26. doi: 10.1212/WNL.0000000000005684

63. Cho AH, Kim HR, Kim W, Yang DW. White matter hyperintensity in ischemic stroke patients: it may regress over time. J Stroke. (2015) 17:60-6. doi: 10.5853 /jos.2015.17.1.60

64. Durand-Birchenall J, Leclercq C, Daouk J, Monet P, Godefroy O, Bugnicourt JM. Attenuation of brain white matter lesions after lacunar stroke. Int J Prev Med. (2012) 3:134-8.

65. Yamada K, Sakai K, Owada K, Mineura K, Nishimura T. Cerebral white matter lesions may be partially reversible in patients with carotid artery stenosis. Am J Neuroradiol. (2010) 31:1350-2. doi: 10.3174/ ajnr.A1873

66. Wardlaw JM, Chappell FM, Valdés Hernández MDC, Makin SDJ, Staals J, Shuler K, et al. White matter hyperintensity reduction and outcomes after minor stroke. Neurology. (2017) 89:1003-10. doi: 10.1212/WNL.0000000000004328

67. Ding $\mathrm{X}, \mathrm{Wu} \mathrm{J}$, Zhou Z, Zheng J. Specific locations within the white matter and cortex are involved in the cognitive impairments associated with periventricular white matter lesions (PWMLs). Behav Brain Res. (2015) 289:9-18. doi: 10.1016/j.bbr.2015.04.021

68. Nolze-Charron G, Mouiha A, Duchesne S, Bocti C, Alzheimer's Disease Neuroimaging Initiative. White matter hyperintensities in mild cognitive impairment and lower risk of cognitive decline. J Alzheimer's Dis. (2015) 46:855-62. doi: 10.3233/JAD-140618

Conflict of Interest Statement: The authors declare that the research was conducted in the absence of any commercial or financial relationships that could be construed as a potential conflict of interest.

Copyright $\odot 2019$ Wu, Ge, Du, Wang, Sun, Han, Ding, Cao, Xu and Zhou. This is an open-access article distributed under the terms of the Creative Commons Attribution License (CC BY). The use, distribution or reproduction in other forums is permitted, provided the original author(s) and the copyright owner(s) are credited and that the original publication in this journal is cited, in accordance with accepted academic practice. No use, distribution or reproduction is permitted which does not comply with these terms. 